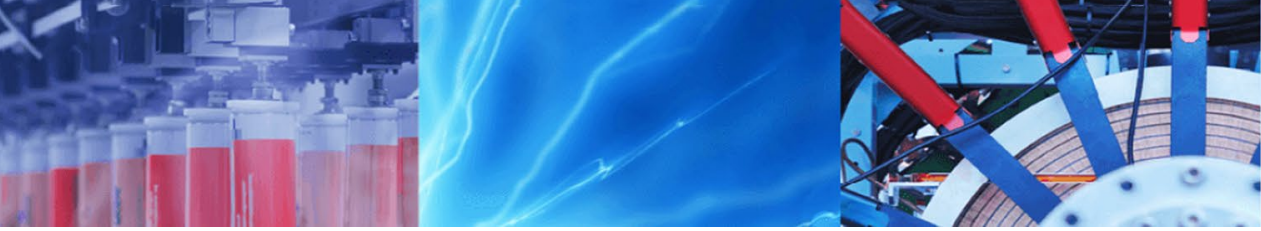

Research Article

\title{
Encapsulation of paraffin wax by rigid cross-linked poly (styrene divinylbenzene-acrylic acid) and its thermal characterization
}

\author{
Ritesh Pradhan ${ }^{1}$ - Arun Prasath Ramaswamy ${ }^{1}$
}

(c) Springer Nature Switzerland AG 2019

\begin{abstract}
The latent heat phase change materials (PCMs) have emerged as an important and effective thermal storage material. The massive change in volume and the requirement for specialized containment of PCMs limits the thermal storage application of the common PCM like paraffin wax. Herein, we report the encapsulation of paraffin wax with the crosslinked poly(styrene-divinylbenzene-acrylic acid) shell via suspension polymerization. The optical and SEM images of the prepared encapsulated phase change materials (EPCMs) were in the size range of 200-500 $\mu \mathrm{m}$. The thermal chargingdischarging rate of the EPCM was studied using a facile water bath technique. It was found that the optimized EPCM showed about 1.7 times faster-charging and 3.5 times faster discharging rate compared to reference PCM (without encapsulation). The latent heat for the EPCM was found to be about 3.3 times higher than the reference PCM. The thermal charging-discharging ability and latent heat values are higher for the encapsulated paraffin wax when compared with reference paraffin wax. The encapsulation of paraffin wax by polymeric materials via facial suspension polymerization using polymerizable vinyl monomers could find its application as heat storage materials.
\end{abstract}

Keywords Phase change materials (PCMs) · Encapsulation · Polymerization · Solar thermal · Latent heat storage · Thermal properties

\section{Introduction}

The solar thermal energy systems have established as a source of the clean thermal energy harvesting technique, -though it suffers from a lack of proper heat storage systems. The solar thermal systems can harness solar energy most efficiently during bright sunny days and the stored thermal energy can be utilized at maximum on as and when required modes, such as during the night or in cold winter or convert solar thermal to electricity [1, 2]. There are various materials have been successfully used to store thermal energy based on sensible heat, latent heat via phase change materials (PCM), and thermo-chemical reaction concepts. Phase change materials (PCM) are emerging as a novel method to facilitate the optimal utilization of the thermal energy systems by providing a heat storage medium $[3,4]$. A phase change material changes its state from solid to liquid state due to gaining of thermal energy and changes from liquid to solid due to release of energy via latent heat storage concept. Phase change materials are categorized into (i) organic-paraffin $\left(\mathrm{C}_{n} \mathrm{H}_{2 n+2}\right)$ and non-paraffin, (ii) inorganic-salt hydrates $\left(\mathrm{M}_{n} \mathrm{H}_{2} \mathrm{O}\right)$ and (iii) eutectic,- - combination of organic and inorganic compounds $[5,6]$. Paraffin waxes are the most common PCM for thermal management having a high heat of fusion per unit weight (i.e. energy density), the selection of PCM depends upon its application in varied thermal ranges, dependable thermal cycling, non-corrosive and chemically inert nature [7]. Even though paraffin wax has its advantages, designing systems with paraffin require greater attention towards (i) void management i.e., due to the volume change from solid to liquid $[2,3,5]$,

$\triangle$ Arun Prasath Ramaswamy, raprasath.get@pondiuni.edu.in | ${ }^{1}$ Laboratory for Energy Materials and Sustainability, Centre for Green Energy Technology, Madanjeet School of Green Energy Technologies, Pondicherry University, Kalapet 605014, India.

SN Applied Sciences (2019) 1:859 | https://doi.org/10.1007/s42452-019-0891-8 
(ii) sub-cooling, and (iii) instability of paraffin wax [1, 2],which can reduce its functionality as a latent heat storage fluid [8]. The encapsulated PCM materials are largely utilized in space heating and cooling systems, integrated with building materials like concrete or paints $[9,10]$. It is also significant for thermal applications like the solar water heater, solar air heater, and solar dryer [11, 12].

The encapsulation of PCM can be carried out with suitable organic/inorganic or hybrid materials by a variety of techniques. The advanced methods like interfacial polymerization, suspension, and emulsion polymerization techniques have to be carried out for micro-encapsulation and nano-encapsulation of PCMs with polymeric materials. The size of the particle formed can be varied from nano-size to micro-size $[5,13]$. In general, polymerization of vinyl monomers such as styrene (St), divinyl-benzene (DVB), acrylic acid (AA), hydroxyethyl methacrylate (HEMA), methyl methacrylate (MMA), polyvinyl chloride (PVC), etc. $[5,9,14-17]$. can be carried out via free-radical or ionic polymerization through thermal or photochemical route. The radical polymerization of vinyl monomers in the presence of PCMs results in the formation of spherical globules of encapsulated PCMs consisting of two parts (i) inner part called core, containing the PCMs and (ii) outer layer called the shell, which is made up of varied polymers or inorganic or hybrid materials to protect the PCMs [9]. Sililuk et al. reported an enhancement in the latent heat of paraffin wax by encapsulating DVB (divinylbenzene)-styrene copolymer. The experimental samples were prepared with $60 \%$ loading of paraffin wax by weight and a varied weight ratio of DVB to styrene. The ratio of DVB and styrene was optimized at the weight ratio of 30:70 to improve the latent heats of the encapsulated paraffin wax with sufficient polymer shell strength. The group reported that the thermal properties of RT27encapsulated with copolymer using DVB and styrene was $\Delta \mathrm{Hm}=153 \mathrm{~kJ} / \mathrm{kg}$ and $\Delta \mathrm{HC}=164 \mathrm{~kJ} / \mathrm{kg}$, which is much higher than those encapsulated using only DVB $(\Delta \mathrm{Hm}=127 \mathrm{~kJ} / \mathrm{kg}$ and $\Delta \mathrm{Hc}=133 \mathrm{~kJ} / \mathrm{kg})$ and closed to bulk RT27 $(\Delta \mathrm{Hm}=162 \mathrm{~kJ} / \mathrm{Kg}$ and $\Delta \mathrm{Hc}=168 \mathrm{~kJ} / \mathrm{kg})$ [5]. Taegu Do et al. reported that the fabrication of hydrophilic poly (2-hydroxyethyl methacrylate) (pHEMA) encapsulated paraffin-wax sphere as the PCM capsule with high encapsulation ratio and thermal storage capability. The thermal conductivity improved from calculated $0.26-0.47 \mathrm{~W} /$ $(\mathrm{m} \cdot \mathrm{K})$ at $25^{\circ} \mathrm{C}$ in comparison with a dry PCM capsule and showed higher durability in the melting-freezing cycle test due to the hydrophilic shell [18]. Ahmed Sari et al. reports that polystyrene based PS/n-heptadecane micro- and nano-capsules were synthesized as novel capsule material using the mini-emulsion polymerization method. The DSC analysis showed that the prepared $\mathrm{n}$-heptadecane/ PS micro/nano-capsules (1:2) with loading of $63.3 \mathrm{wt} \%$ of $n$-heptadecane content had a melting temperature of $21.48^{\circ} \mathrm{C}$ and latent heat of $136.89 \mathrm{~kJ} / \mathrm{kg}$, and a sub-cooling degree as little as $0.11^{\circ} \mathrm{C}$ making encapsulated PCM useful for temperature regulation application [16]. It has been reported that the encapsulated PCM showed better thermal efficiency for the prepared samples via suspension polymerization [5]. Over a decade, several researchers have reported that the encapsulation of PCMs by suspension polymerization using various monomers to have varied polymer shells. For example, microencapsulation of paraffin wax with poly(styrene-methylmethacrylate), poly(methylmethacrylate-methacrylic acid), polystyrene, polymethylmethacrylate [PMMA] with different crosslinking agents and poly-divinylbenzene were carried out by suspension polymerization $[19,20]$. This paper reports the encapsulation of paraffin wax (having 31 carbon chain commonly used as an organic PCM material) by microencapsulation to create highly thermally stable PCM via suspension polymerization. The encapsulated PCM was synthesized via the free-radical suspension polymerization process with the varying weight ratio of monomers (di-vinyl benzene, styrene, and acrylic acid) to produce micro-sized encapsulated PCM spheres for better stability and higher thermal performance. In addition, this work highlights the performance analysis of encapsulated PCM compared with pure paraffin wax with regard to thermophysical properties. The optimum increase in cross-linking density in the encapsulated PCM showed an increase in the thermal properties. This study clearly outlines that the thermal charging-discharging ability and latent heat value have considerably improved for the encapsulated paraffin wax compared to pure un-encapsulated paraffin wax.

\section{Experimental method}

\subsection{Materials}

The laboratory grade chemicals paraffin wax, di-vinyl-benzene (DVB), styrene (St), acrylic acid (AA) and potassium persulfate (KPS) were purchased from the Sigma Aldrich. The monomers St and DVB were purified by standard methods to remove the impurities [5, 14]. The glassware required for the experimental set-up was washed using DI water, IPA and dried before use. The paraffin wax was taken as the phase change material with a melting point of $56.06{ }^{\circ} \mathrm{C}$ and latent heat of fusion of $151.61 \mathrm{~kJ} / \mathrm{kg}$ as observed from the DSC curve.

\subsection{Synthesis of encapsulated PCMs}

The encapsulation of PCM was carried out by suspension free-radical polymerization technique. Table 1 depicts the recipe for the various encapsulated phase change 
Table 1 Recipe for the synthesis of encapsulated paraffin wax using monomers St, DVB, and AA by free-radical suspension polymerization ${ }^{a}$

\begin{tabular}{|c|c|c|c|c|c|c|c|c|c|}
\hline Sample No. & Monomers & Wax (g) & Water (ml) & DVB (g) & St (g) & $\mathrm{AA}(\mathrm{g})$ & KPS (mg) & $\begin{array}{l}\text { Wax to monomer } \\
\text { ratio }(\mathrm{Wt})\end{array}$ & Yield (\%) \\
\hline EPCM1 & DVB:AA & 6.5 & 150 & 14.62 & Nil & 8.4 & 230 & $22: 78$ & 95 \\
\hline EPCM2 & DVB:AA:ST & 6.5 & 150 & 12.79 & 1.8 & 8.4 & 230 & $28: 72$ & 94 \\
\hline EPCM3 & DVB:AA:ST & 6.5 & 150 & 10.96 & 3.6 & 8.4 & 230 & $28: 72$ & 96 \\
\hline EPCM4 & DVB:AA:ST & 6.5 & 150 & 9.14 & 5.4 & 8.4 & 230 & $28: 72$ & 95 \\
\hline EPCM5 & DVB:AA:ST & 6.5 & 150 & 7.31 & 7.2 & 8.4 & 230 & $28: 72$ & 97 \\
\hline EPCM6 & DVB:AA:ST & 6.5 & 150 & 7.31 & 7.2 & 8.4 & 230 & $28: 72$ & 96 \\
\hline EPCM7 & DVB:ST & 6.5 & 150 & 9.14 & 5.4 & Nil & 230 & $31: 69$ & 98 \\
\hline EPCM8 & DVB:ST & 6.5 & 150 & 10.96 & 3.6 & Nil & 230 & $31: 69$ & 94 \\
\hline EPCM9 & DVB:ST & 6.5 & 150 & 12.79 & 1.8 & Nil & 230 & $31: 69$ & 95 \\
\hline EPCM10 & DVB:ST & 6.5 & 150 & 7.31 & 7.2 & Nil & 230 & $31: 69$ & 97 \\
\hline
\end{tabular}

${ }^{\mathrm{a}}$ Reaction time $6 \mathrm{~h}$ for all the samples

materials (EPCMs) prepared. The suspension polymerization for the encapsulation of paraffin wax by cross-linked polymer network was carried out with the weight-based ratios of paraffin wax to monomer ratio as 31:69, 28:72 and 22:78. The monomers (St and DVB with/without $A A)$ were mixed in a flask and degassed with nitrogen for $\sim 15$ min to remove oxygen. The initiator KPS was dissolved in water and degassed for $\sim 10 \mathrm{~min}$. Paraffin wax was melted in an RB flask placed at $70^{\circ} \mathrm{C}$ using silicon oil bath. The DI water of $150 \mathrm{ml}$ degassed with nitrogen (to remove oxygen) for $\sim 25 \mathrm{~min}$ and added to the melted wax with continuously stirring at $200 \mathrm{rpm}$ for $\sim 15 \mathrm{~min}$ using a mechanical stirrer for effective mixing. Finally, the monomers (DVB and Styrene) along with KPS were added to the melted paraffin wax in water. The resulting suspension was stirred for $8 \mathrm{~h}$ at the stirring speed of about $500 \mathrm{rpm}$ by maintaining the reaction temperature at $70^{\circ} \mathrm{C}$ to complete the polymerization reaction under a nitrogen atmosphere. Figure 1 shows the schematic synthesis route of various encapsulated PCM microcapsules carried for this work.

\subsection{Characterization}

The optical image microscope and Scanning Electron Microscope (SEM) were used to study the morphology of the as-synthesized encapsulated PCMs. The samples were dispersed in water before analyzing with an optical microscope. For SEM analysis, the samples were dispersed in a graphite grid for analyzing. Thermal characterization of the selected encapsulated PCMs was carried out using (i) Water Bath Technique, (ii) Thermal Gravimetric Analysis (TGA) and (iii) Differential Scanning Calorimetry (DSC). The water bath technique has been meant to work on the basis of heat transfer between water and the PCMs. The comparative analysis of various encapsulated PCMs and paraffin wax for thermal charge and discharge with time was studied using water bath technique set-up made in our laboratory with temperature sensors. A fixed amount of paraffin wax or EPCMs placed inside three testing tubes and the same was placed inside the heated water bath. The temperature sensors with data logger have been used to sense the temperature of wax and water bath until temperature reaches the equilibrium stage. The melted wax is
Fig. 1 Schematic illustration of the synthesis of microencapsulation of organic PCM paraffin wax

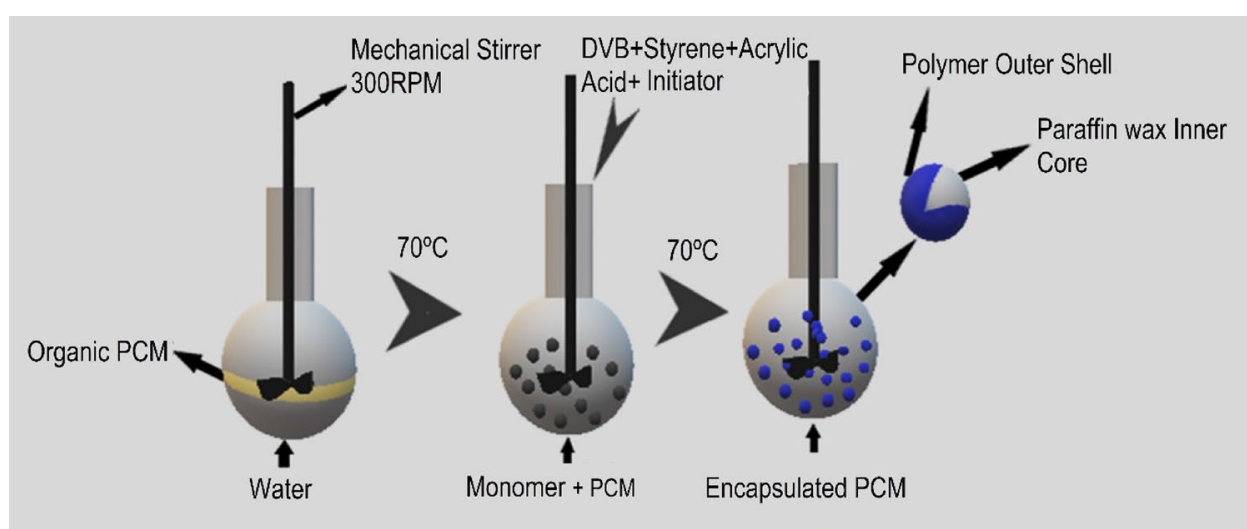


then placed in the water bath at room temperature during which the liquid phase wax converts to solid. The change in temperature is noted using data logger throughout the experiment. Figure 2 shows the experimental set-up to investigate the thermal charging and discharging using the water bath technique. Similar experiments were carried out for the different samples and a temperature-time graph plotted to compare paraffin wax and encapsulated phase change materials.

The TGA analysis was carried out at the heating rate of $5^{\circ} \mathrm{C} / \mathrm{min}$ from room temperature to $800^{\circ} \mathrm{C}$ in a nitrogen atmosphere to investigate the thermal stability of the selected EPCMs and paraffin wax. The thermal study provides information about the change in physical properties like phase transitions, absorption, desorption, chemisorption, thermal stability, and solid-gas reaction $[6,15]$. The Differential scanning calorimeter (DSC) was used to measure the phase change properties of paraffin wax and selected samples of EPCMs. The DSC was carried out in the temperature range of $30-70^{\circ} \mathrm{C}$ at a heating/cooling rate of $5^{\circ} \mathrm{C} / \mathrm{min}$ in a nitrogen atmosphere to evaluate the phasechange behaviors $[14,16]$. The percentage of paraffin wax loading in the encapsulated PCM was calculated using the below equation

The percentage of paraffin wax loading $=\frac{\Delta H_{\text {ecapsulatedPCM }}}{\Delta H_{\text {paraffin }}} \times 100$

where $\Delta \mathrm{H}_{\text {encapsulatedPCM }}$ and $\Delta \mathrm{H}_{\text {paraffin }}$ are the measured melting enthalpy as per report [8].

\section{Results and discussion}

The encapsulation of paraffin wax was carried out by batch suspension free-radical polymerization using the varied weight ratio of paraffin wax to vinyl monomers (DVB/St/
AA) as depicted in Table 1. The yield of the EPCMs was analyzed by the gravimetric method, which revealed that the conversions were in the range of $94-97 \%$. Figure 3 shows the optical image of the encapsulated PCM samples observed at the magnification of $1000 \times$ and $15.5 \mu \mathrm{m}$ depth of field. The image shows spherical as well as irregular shape/size structure in the range $\sim 100-600 \mu \mathrm{m}$ for the observed samples of EPCM7 and EPCM10.

Figure 4 shows the representative SEM image of EPCM7 and EPCM10 prepared with varied DVB: St weight ratio. The size of the encapsulated phase change material was found to be in the range of $\sim 200-400 \mu \mathrm{m}$ with sphericallike as well as irregular shapes. The wide range of size observed from SEM images indicates that the EPCMs of varied sizes were formed and opens the scope for reaction optimization to produce the required controlled size particles. Figure 5 shows the temperature-time graph plotted using the data derived from thermal charging and discharging analysis during heating and cooling of paraffin wax and EPCMs with temperature change. In the case of the EPCMs, the cross-linked polymer shell in the sample contributes to the heat transfer and affects the heat storage capacity of the EPCMs. The shell material of poor thermal conductivity can seriously depreciate the heat storing ability of the EPCMs. However, it is observed from the heating graph that the EPCM7 (DVB: ST; the weight-based ratio of 1.69) without using acrylic acid showed 1.7 times faster temperature rise than blank paraffin wax and also stabilizes at $10^{\circ} \mathrm{C}$ higher temperature. The other samples also showed better thermal charging and discharging than paraffin wax based on the varied weight ratio of DVB:St:AA. Thus, we conclude that the weight ratio of monomers in the polymer and the composition of the polymer influence the thermal properties (thermal charging and discharging ability) of the EPCMs. The temperature-time graph (Fig. 5b) shows the plot using the data from the cooling experiment for the samples in the water bath. From the cooling graph,
Fig. 2 Experimental set-up to investigate the thermal charging and discharging using water bath technique

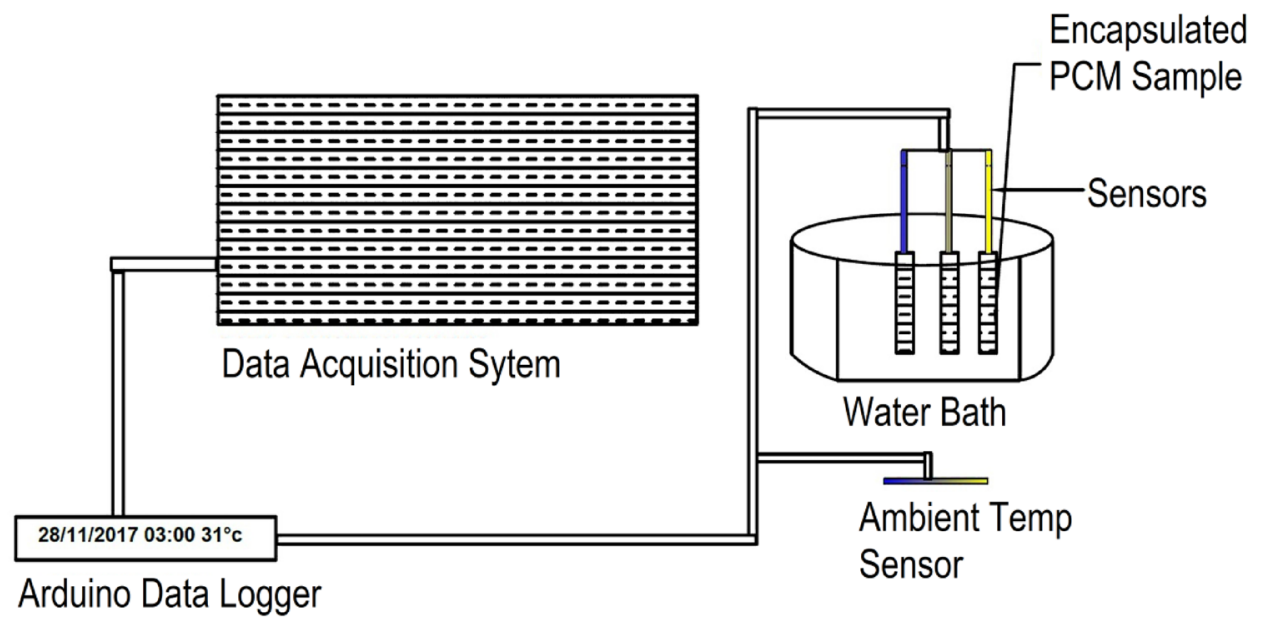



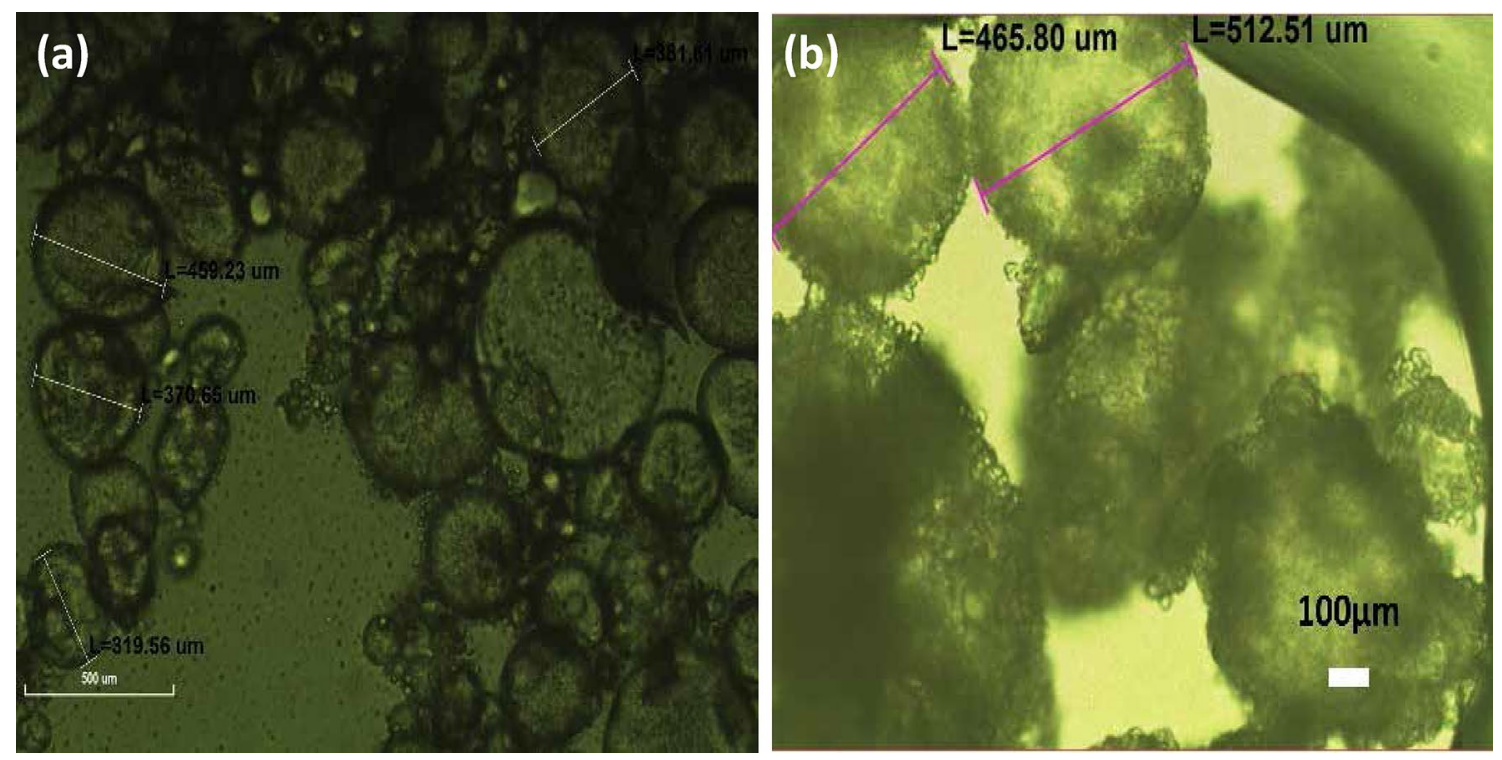

Fig. 3 Optical image of encapsulated PCM prepared by radical polymerization with a variable composition of monomers, a EPCM7 and $\mathbf{b}$ EPCM10
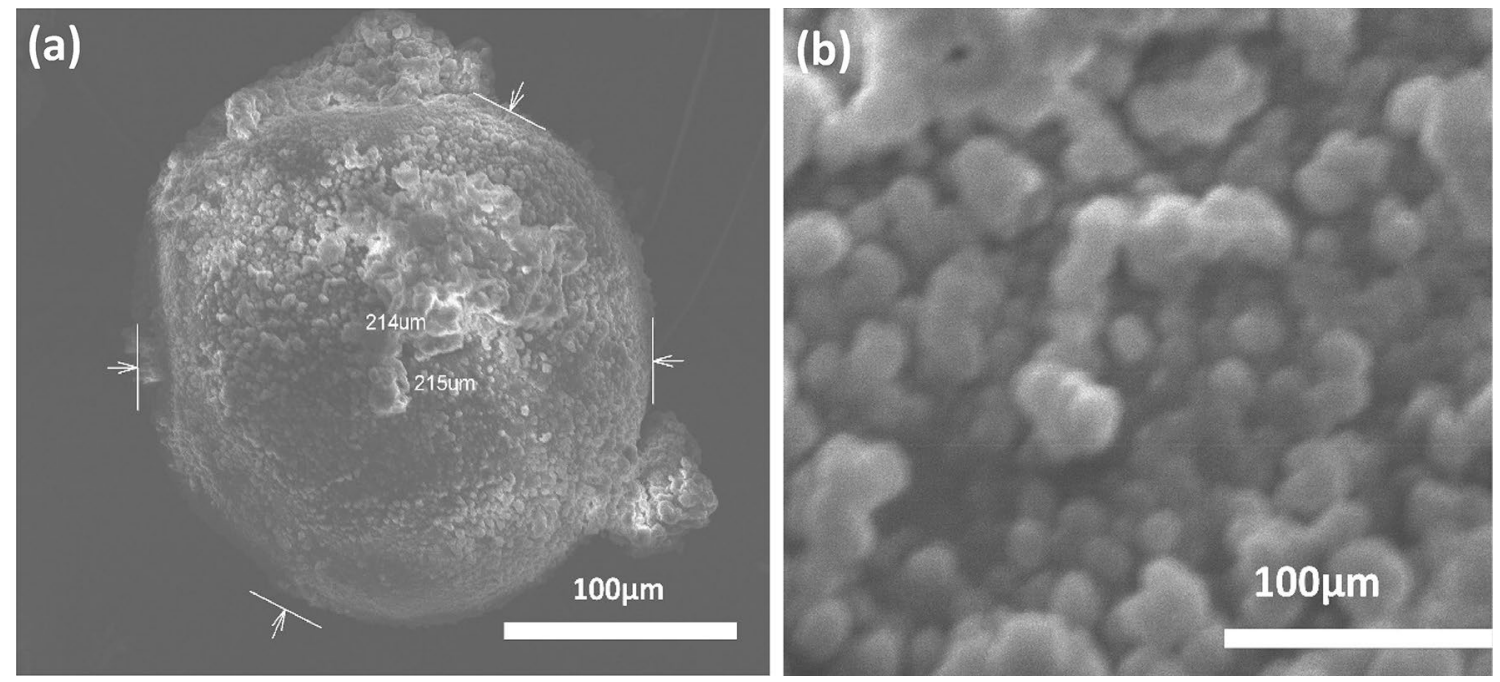

Fig. 4 SEM image of encapsulated PCM prepared by using a various weight ratio of DVB and Styrene a EPCM7 and b EPCM10

it is observed that the temperature decrement was highest when the paraffin wax is encapsulated with DVB: St (at the weight base ratio of 1.69). From the water bath technique, we observed a faster charging and discharging temperature for encapsulated sample EPCM7. The sample, EPCM7 has higher crosslinking network compared to EPCM10 due to a higher amount of DVB. In addition, the charging and discharging data of EPCM1 and EPCM2 samples were slower compared to EPCM7,- -this could be attributed for lower crosslinking network in the samples due to hydrophilic acrylic monomer (a non-crosslinking monomer). Thus, we believe that the increase in the crosslinking density and in-turn increased the rate of charging and discharging, -thus DVB plays a vital role in heat transfer in EPCMs.

The TG curve showed in Fig. 6 for the dried encapsulated PCM with DVB: St (weight-based ratio of 1.69) showed the thermal decomposition of paraffin wax $\left(180^{\circ} \mathrm{C}\right)$ and of the polymer $\left(500^{\circ} \mathrm{C}\right)$ in two respective steps. The complete volatilization/decomposition was observed for bulk paraffin wax at $250^{\circ} \mathrm{C}$. This result confirmed the existence of paraffin wax in the prepared capsules. The slight difference in the decomposition curve between bulk paraffin wax and the encapsulated paraffin wax can be attributed to the 

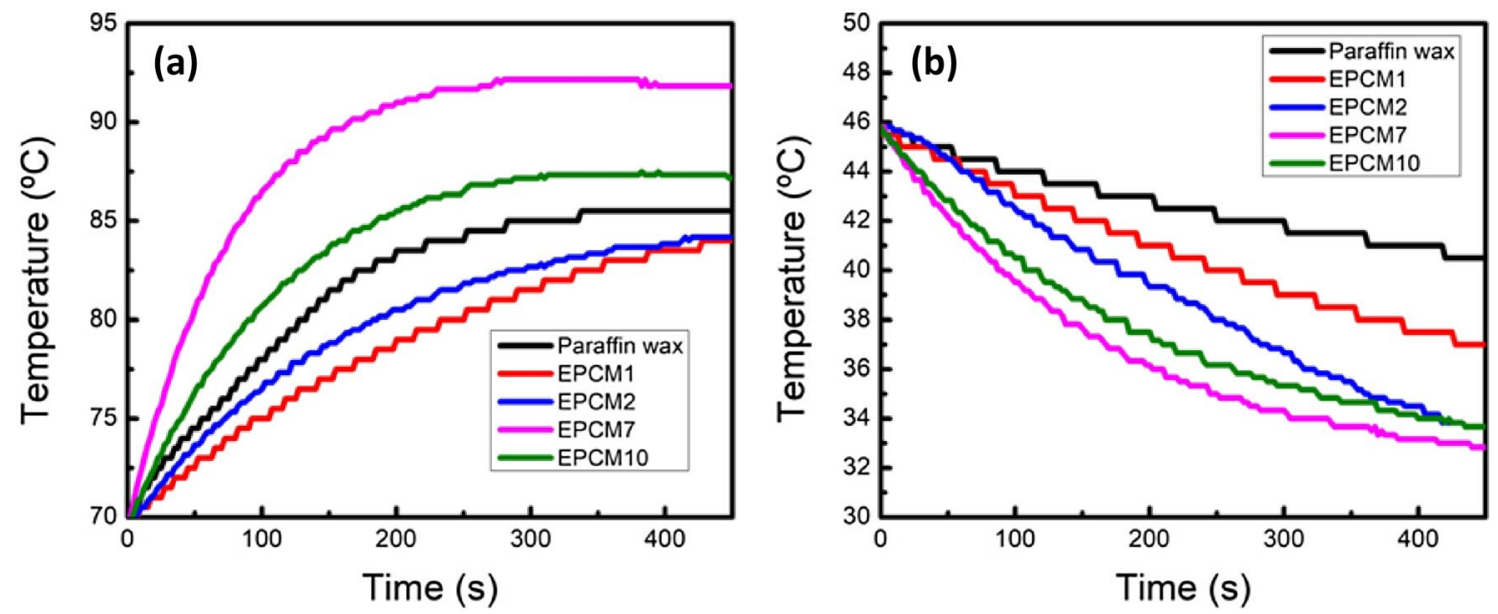

Fig. 5 Graph showing the temperature-time curve of encapsulated PCM material for $\mathbf{a}$ heating cycle and $\mathbf{b}$ cooling cycle

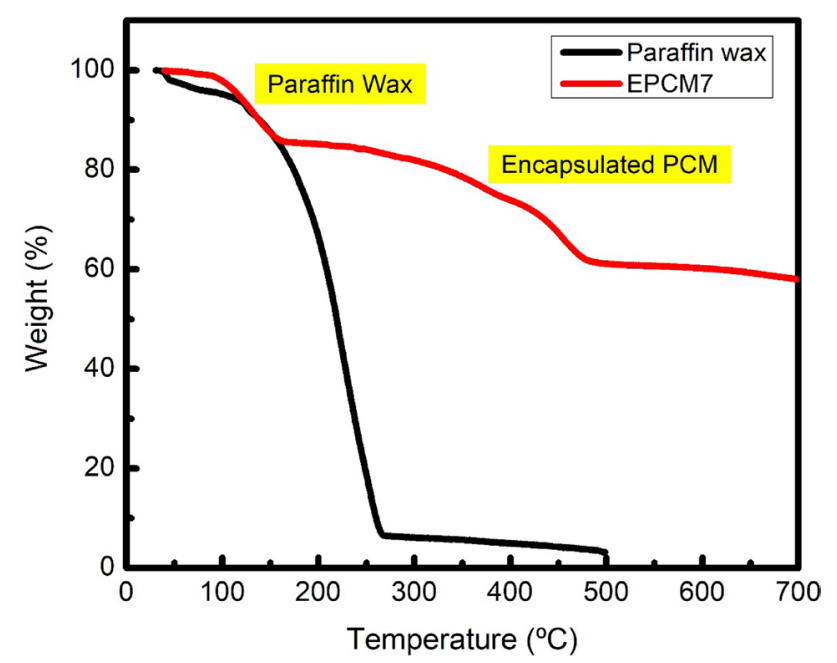

Fig. 6 TGA curve of the sample EPCM7 and blank paraffin wax

effect of the polymeric shell/matrix present. As an equal mass of paraffin wax and encapsulated PCM was taken for the characterization, the decrease in the decomposition temperature range for paraffin wax was due to lower mass of paraffin wax in the composite structure. From the TG curve (Fig. 6), it can be concluded that for the same temperature range, the mass volatilization/decomposition of paraffin wax occurs rapidly as compared to encapsulated PCM.

The results from differential scanning calorimetry analyzed for pure wax and selected EPCMs are shown in Fig. 7 and the derived data have been summarized in Table 2 . The DSC curve of pure paraffin wax displayed well-defined two endothermic peaks at $41.02^{\circ} \mathrm{C}$ at $56.06{ }^{\circ} \mathrm{C}$ [21]. The first minor peak at $41.02^{\circ} \mathrm{C}$ corresponds to the solid-solid phase transition of paraffin $\left(T_{t}\right)$ and the second major peak

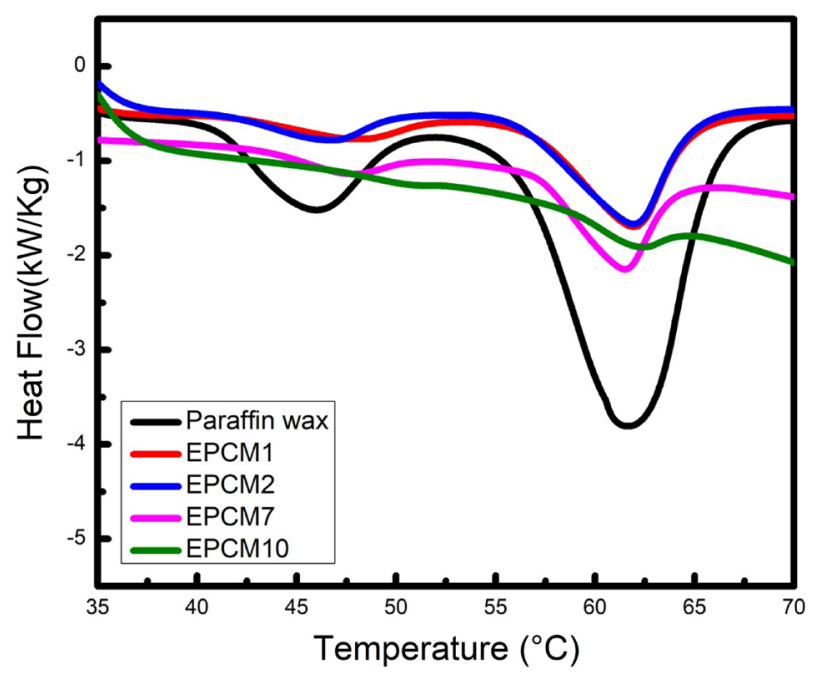

Fig. 7 DSC curve of the blank paraffin wax and selected EPCMs displayed well-defined two endothermic peaks

at $56.06{ }^{\circ} \mathrm{C}$ correspond to the solid-liquid phase change of the paraffin $\left(T_{m}\right)$. The latent heat of the solid-liquid (LHS-L) transition of pure paraffin wax was found to be $151.62 \mathrm{~kJ} /$ kg. However, the EPCMs showed a slight increase in endothermic melting peaks $\left(T_{t} / T_{m}\right)$ with respect to monomers styrene and divinylbenzene ratio as depicts in Table 2 . The increases in the cross-linking density due to higher concentration divinylbenzene in the EPCMs can subsequently increase in the endothermic melting peaks due to high compactness. The endothermic peaks $\left(T_{t} / T_{m}\right)$ for the EPCMs seen to vary with the composition of paraffin wax, vinyl monomers and its cross-linking density due to divinylbenzene ratio to styrene, -which suggests that the encapsulation of paraffin wax with a varied weight ratio of monomers have a significant effect in the solid-solid and 
Table 2 DSC data analysis for paraffin wax and selected EPCMs prepared at a varied monomer weight ratio

\begin{tabular}{|c|c|c|c|c|c|c|}
\hline \multirow[b]{2}{*}{ Sample no } & \multicolumn{2}{|c|}{ Monomer ratio } & \multicolumn{2}{|c|}{ Melting process } & \multirow[b]{2}{*}{$\Delta \mathrm{H}_{\text {melting }}(\mathrm{kJ} / \mathrm{kg})$} & \multirow[b]{2}{*}{ Core\% } \\
\hline & DVB: ST & $A A(g)$ & $\mathrm{T}_{\mathrm{t}}\left({ }^{\circ} \mathrm{C}\right)$ & $\mathrm{T}_{\mathrm{m}}\left({ }^{\circ} \mathrm{C}\right)$ & & \\
\hline EPCM1 & $100: 0$ & 8.4 & 41.91 & 56.70 & 56.79 & 37.46 \\
\hline EPCM2 & $88: 12$ & 8.4 & 41.35 & 55.90 & 62.36 & 41.13 \\
\hline EPCM7 & $63: 37$ & 8.4 & 43.64 & 57.27 & 39.78 & 26.24 \\
\hline EPCM10 & $50: 50$ & 8.4 & 47.46 & 58.92 & 13.94 & 9.19 \\
\hline Paraffin wax & - & - & 41.02 & 56.06 & 151.62 & - \\
\hline
\end{tabular}

solid-liquid phase transition $\left(T_{t} / T_{m}\right)$ of the core material. The percentage of paraffin wax inside the polymer shell represented the effective performance of the PCM for latent heat storage purposes. The latent heat of the pure paraffin wax measured from DSC was $151.62 \mathrm{~kJ} / \mathrm{kg}$. The paraffin wax weight percentage value calculated from the DSC results for various EPCMs using the standard equation [8], showed in the range of 9-41\% (Table 2). From the data, it is very clear that higher paraffin wax as core $(41.13 \%)$ for the EPCM2 shows lower solid-solid phase transition $\left(T_{t}\right)$ at $41.35^{\circ} \mathrm{C}$ and solid-liquid phase change $\left(\mathrm{T}_{\mathrm{m}}\right)$ at $55.90^{\circ} \mathrm{C}$ with latent heat fusion measured from DSC as $62.36 \mathrm{~kJ} /$ $\mathrm{kg}$. Whereas, the EPCM10 with lowest paraffin wax as core (9.19\%) showed higher solid-solid phase transition $\left(T_{t}\right)$ at $47.46{ }^{\circ} \mathrm{C}$ and solid-liquid phase change $\left(T_{m}\right)$ at $58.92{ }^{\circ} \mathrm{C}$ with lowest latent heat fusion measured from DSC as $13.94 \mathrm{~kJ} / \mathrm{kg}$. However, the overall latent heat of the material increases due to the presence of the high amount of paraffin wax and the cross-linked density in the polymeric shell. The DSC results indicated that even though the paraffin material decreased with a high amount of polymer layer, -the microcapsules could perform the energy storage-release function at a higher temperature for EPCMs without leaks compare to pure paraffin wax and depend upon the type and nature of the polymeric network.

\section{Conclusion}

The encapsulation of paraffin wax via free-radical suspension polymerization of monomers such as divinylbenzene and styrene and acrylic acid at a varied weight ratio of monomers (DVB:St) resulted in the microcapsules of sizes $200-500 \mu \mathrm{m}$. The thermal analysis of the encapsulated PCM showed charging and discharging rate of the encapsulated PCM increased resulting in a faster thermal response. This rapid heating and the cooling feature of EPCMs has significant importance in achieving higher heat transfer in thermal energy systems. The latent heat of the EPCMs varied with respect to weight ratio of paraffin wax to polymer shell structure, and by varying the monomers composition. The loading of paraffin wax in EPCM can be optimized by changing the reaction parameters (e.g. reaction time and temperature, wax to monomer ratio, and paraffin wax nature) and hence latent heat can be enhanced or influenced. It can be concluded that the encapsulation of paraffin wax by polymeric shell can produces favorable influences on improvising the thermal properties of paraffin composite material. Thus, the freeradical suspension polymerization technique using vinyl monomers of styrene and divinylbenzene, and acrylic acid (DVB-ST-AA co-polymer) was found to be an efficient method to encapsulate the paraffin wax. This research work opens up a new direction, where the PCM materials can be modified to specifically applicable to produce EPCMs with desired physical and thermal properties.

Acknowledgements One of the authors R.P is grateful to SAF foundation for supporting his PG study with fellowship. We acknowledge CIF, Pondicherry University for thermal analysis and Dr. P.Thangadurai, CNST, Pondicherry University for optical images.

\section{Compliance with ethical standards}

Conflict of interest On behalf of all authors, the corresponding author states that there is no conflict of interest.

\section{References}

1. Alva G, Liu L, Huang X, Fang G (2017) Thermal energy storage materials and systems for solar energy applications. Renew Sustain Energy Rev 68:693-706

2. Salunkhe PB, Shembekar PS (2012) A review on effect of phase change material encapsulation on the thermal performance of a system. Renew Sustain Energy Rev 16:5603-5616

3. Jamekhorshid A, Sadrameli SM, Farid M (2014) A review of microencapsulation methods of phase change materials (PCMs) as a thermal energy storage (TES) medium. Renew Sustain Energy Rev 31:531-542

4. Kant K, Shukla A, Sharma A (2017) Advancement in phase change materials for thermal energy storage applications. Sol Energy Mater Sol Cells 172:82-92

5. Namwong S, Noppalit S, Okubo M et al (2015) Latent heat enhancement of paraffin wax in poly (divinylbenzene-comethyl methacrylate) microcapsule. Polym Plast Technol Eng 54:779-785

6. Hoang HM, Leducq D, Perez-Masia R et al (2015) Heat transfer study of submicro-encapsulated PCM plate for food packaging application. Int J Refrig 52:151-160 
7. Wei NTJ, Nan WJ, Guiping C (2017) Experimental study of efficiency of solar panel by phase change material cooling. In: IOP conference series: materials science and engineering. IOP Publishing, $\mathrm{p} 12011$

8. Gao X, Zhao T, Luo G et al (2018) Facile method of fabricating microencapsulated phase change materials with compact bonding polymer-silica hybrid shell using TEOS/MPS. Thermochim Acta 659:183-190

9. Chen F, Wolcott M (2015) Polyethylene/paraffin binary composites for phase change material energy storage in building: a morphology, thermal properties, and paraffin leakage study. Sol Energy Mater Sol Cells 137:79-85

10. Cui Y, Xie J, Liu J et al (2017) A review on phase change material application in building. Adv Mech Eng 9:1687814017700828

11. Shamsi H, Boroushaki M, Geraei H (2017) Performance evaluation and optimization of encapsulated cascade PCM thermal storage. J Energy Storage 11:64-75

12. Johansen JB, Englmair $G$, Dannemand $M$ et al (2016) Laboratory testing of solar combi system with compact long term PCM heat storage. Energy Procedia 91:330-337

13. Nath R (2012) Encapsulation of high temperature phase change materials for thermal energy storage. University of South Florida, Tampa

14. Mochane MJ, Luyt AS (2012) Preparation and properties of polystyrene encapsulated paraffin wax as possible phase change material in a polypropylene matrix. Thermochim Acta 544:63-70

15. Ng D-Q, Tseng Y-L, Shih Y-F et al (2017) Synthesis of novel phase change material microcapsule and its application. Polym (Guildf) 133:250-262
16. Sarı A, Alkan C, Döğüşcü DK, Biçer A (2014) Micro/nano-encapsulated $\mathrm{n}$-heptadecane with polystyrene shell for latent heat thermal energy storage. Sol Energy Mater Sol Cells 126:42-50

17. Wang Y, Shi H, Xia TD et al (2012) Fabrication and performances of microencapsulated paraffin composites with polymethylmethacrylate shell based on ultraviolet irradiation-initiated. Mater Chem Phys 135:181-187

18. Do T, Ko YG, Chun Y, Choi US (2015) Encapsulation of phase change material with water-absorbable shell for thermal energy storage. ACS Sustain Chem Eng 3:2874-2881

19. Sánchez Silva L, Rodríguez JF, Romero A, Sánchez P (2012) Preparation of coated thermo- regulating textiles using Rubitherm RT31 microcapsules. J Appl Polym Sci 124:4809-4818

20. Yafei A, Yong J, Jing S, Deqing W (2007) Microencapsulation of $\mathrm{n}$-hexadecane as phase change material by suspension polymerization. e-Polymers 098:1-9

21. Rao ZH, Zhang GQ (2011) Thermal properties of paraffin waxbased composites containing graphite. Energy Sources Part A 33:587-593

Publisher's Note Springer Nature remains neutral with regard to jurisdictional claims in published maps and institutional affiliations. 